\title{
一步电沉积制备交联型有机-无机生物杂化膜构筑 双酶传感器的研究
}

\author{
李峰*，王振，冯艳
}

生态化工教育部重点实验室, 青岛科技大学化学与分子工程学院, 青岛 266042

* 通讯作者, E-mail: lifeng@qust.edu.cn

收稿日期：2009-04-21；接受日期：2009-05-05

摘要利用一步电沉积法, 以含有环氧基团的 $\gamma$-环氧丙氧丙基三甲氧基硅烷 (GPTMS)为无机杂化试剂和功能性交联试剂, 通过壳聚糖(Chitosan, CS)、辣根过 氧化物酶(HRP)和葡萄糖氧化酶(GOD)分子中 $-\mathrm{NH}_{2}$ 与环氧基团的反应，在金电极 表面原位制备交联型有机-无机生物杂化膜, 得到共固定HRP和GOD的新型双酶 生物传感器. 实验证实了这种有机-无机生物杂化膜在不同酸、碱条件下都具有 高的稳定性和耐用性, 克服了CS酸溶的不足, 从而扩大了其使用范围。在葡萄 糖检测中, 交联型双酶传感器HRP-GOD/GPTMS/CS/Au比无交联的双酶传 感器 HRP-GOD/CS/Au 具有更高的灵敏度、更宽的线性范围, 其线性范围为 $1 \mu \mathrm{mol} / \mathrm{L} \sim 351 \mu \mathrm{mol} / \mathrm{L}$, 检测限为 $0.3 \mu \mathrm{mol} / \mathrm{L}$.

关键词

壳聚糖

$\gamma$-环氧丙氧丙基三甲氧基硅烷

葡萄糖

生物传感器

\section{1 引言}

通过两种或多种材料的功能复合、性能互补和优 化, 制备性能优良的杂化材料是现代材料发展的趋 势. 有机-无机杂化材料是杂化材料的一个新领域, 兼具无机材料高的稳定性和有机材料的功能性, 具 有巨大的应用潜力 ${ }^{[1]}$.

壳聚糖(聚(1,4)-2-氨基-2-脱氧- $\beta$-D-葡聚糖，Chitosan, CS), 是天然类多糖甲壳素经部分脱乙酰化后 得到的直链大分子生物多糖. 作为来源丰富、廉价易 得的天然高分子材料, CS 具有良好的生物相容性. 此 外, CS 分子中丰富的氨基和羟基使其具有较强的亲 水性, 并易于衍生化 ${ }^{[2 \sim 5]}$. 将 CS 材料用于构筑生物传 感器的研究非常活跃. 然而, 壳聚糖易溶解于酸或酸 性水溶液, 这在很大程度上限制了 $\mathrm{CS}$ 材料的推广应 用 ${ }^{[5]}$.
将无机材料的稳定性和机械强度引入到 CS中, 制备有机-无机杂化材料是近年来研究的热点. 最近, 基于壳聚糖的有机-无机杂化材料已被作为功能性生 物材料广泛应用于酶的固定化 ${ }^{[6-8]}$. Wang等 ${ }^{[6]}$ 将壳聚 糖与四乙氧基硅烷进行杂化, 形成一种多孔的有机一 无机杂化材料，并用于玻碳电极表面HRP的固定化. Liu和 shirosaki等 ${ }^{[011]}$ 利用 CS 与含有环氧基团的 $\gamma$-环 氧丙氧丙基三甲氧基硅烷(GPTMS)的共价反应，通 过溶胶-凝胶反应制备了交联型有机-无机杂化材料. 在上述溶胶-凝胶过程中CS的共价交联与无机网络的 引入一步完成： $\operatorname{CS}$ 链上的氨基与硅氧烷分子中的环 氧基团共价结合，同时硅氧烷水解、缩合形成无机网 络结构. 所得交联型有机-无机杂化材料具有高的稳 定性和生物相容性.

CS 电沉积法基于 CS 结构特点和电化学原理, 利 
用电解水过程中阴电极周围形成的 $\mathrm{pH}$ 梯度原位沉积 CS 膜. 自陈洪渊等 ${ }^{[12 ~ 14]}$ 提出将电沉积法作为制备多 组分壳聚糖复合膜的普适方法以来, CS 电沉积法已 成为制备组分、厚度可控的壳聚糖生物复合膜的有效 方法. 利用 CS 电沉积法制备交联型壳聚糖有机-无机 生物杂化膜, 一步共价固定 HRP 和 GOD 构筑 HRP-GOD 双酶传感器的研究尚未见报道.

本文利用一步电沉积法, 以含有环氧基团的 GPTMS 为无机杂化试剂和功能性交联试剂, 通过 CS、HRP和GOD分子中- $\mathrm{NH}_{2}$ 与环氧基团的反应，在金 电极表面原位制备交联型有机-无机生物杂化膜，得 到共固定HRP和GOD的新型双酶生物传感器. 实验 证实了所得的有机-无机生物杂化膜可克服 CS酸溶的 不足, 同时制备的双酶生物传感器具有良好的重现 性和较宽的线性范围.

\section{2 实验部分}

\section{1 仪器与试剂}

仪器: CHI 832B电化学分析仪与CHI 660C电化 学分析仪(上海辰华仪器公司); 金电极及其修饰电极 为工作电极, 铂丝电极为对电极, $\mathrm{Ag} / \mathrm{AgCl}$ 电极为参 比电极; pHS-25 型 $\mathrm{pH}$ 计.

试剂: CS(脱乙酰度 $98 \%$, 平均摩尔分子量 $6 \times$ $10^{4} \mathrm{~g} / \mathrm{mol}$, 中国远华生物医药公司); GPTMS(Alfa aesar); $\mathrm{HRP}(\mathrm{EC}$ 1.11.1.7, $\mathrm{RZ}>3.0,237 \mathrm{U} / \mathrm{mg})$ 与 $\mathrm{GOD}(\mathrm{EC}$ 1.1.3.4, $905 \mathrm{U} / \mathrm{mg}$ ) 购自上海三杰生物有限 公司; $\beta$ - D-葡萄糖(沈阳试剂一厂); 其他试剂均为国 产分析纯, 实验用水为二次蒸馏水.

\section{2 电沉积溶液的制备}

$\mathrm{CS}(\mathrm{pH}$ 5.5)溶液的制备参照文献[12]. 具体为: 将 $\mathrm{CS}$ 粉末加入二次蒸馏水中, 在不断摚拌的情况下, 滴加稀 $\mathrm{HCl}$ 溶液使 $\mathrm{CS}$ 溶解, 此时溶液 $\mathrm{pH}$ 值为 3.0 左右, 加入 $1.0 \mathrm{~mol} / \mathrm{L} \mathrm{NaOH}$ 将溶液调至 $\mathrm{pH} 5.5$.

将一定量的 GPTMS 加入到 CS 溶液中, 超声 5 $\min$ 后, 室温静置 $12 \mathrm{~h}$. 向 GPTMS/CS 溶液加入 HRP 和 GOD, 使电沉积溶液 HRP-GOD/GPTMS/CS 中 HRP 和 GOD 的含量均为 $1 \mathrm{mg} / \mathrm{mL}$.

\section{3 双酶生物传感器的制备}

将裸 $\mathrm{Au}$ 电极在 $30 \% \mathrm{H}_{2} \mathrm{O}_{2}$ 与浓硫酸的混合液
(3: $7 \mathrm{~V} / \mathrm{V}$ )中浸泡 $30 \mathrm{~min}$, 依次用 $1.0,0.3$ 和 $0.05 \mu \mathrm{m}$ $\alpha-\mathrm{Al}_{2} \mathrm{O}_{3}$ 抛光后, 分别在二次蒸馏水与乙醇溶液中超 声清洗. 将处理后的电极浸入到HRP-GOD/GPTMS/ $\mathrm{CS}$ 电沉积溶液中, 在 $-1.0 \mathrm{~V}$ 恒电位下电沉积 $8 \mathrm{~min}$, 即制得HRP-GOD/GPTMS/CS/Au电极. 将电极在 $0.1 \mathrm{~mol} / \mathrm{L}$ 磷酸盐缓冲溶液(PBS, pH 7.0)中清洗, 使用 前于 $4^{\circ} \mathrm{C}$ 下放置保存 $12 \mathrm{~h}^{[13]}$.

\section{4 电化学测定}

将各修饰电极置于 $1.0 \mathrm{mmol} / \mathrm{L} \mathrm{K}_{4}\left[\mathrm{Fe}(\mathrm{CN})_{6}\right]+0.1$ $\mathrm{mol} / \mathrm{L} \mathrm{KCl}$ 混合溶液中, 用 $\mathrm{CHI}$ 832B电化学分析仪记 录循环伏安曲线, 扫描速度为 $50 \mathrm{mV} / \mathrm{s}$; 测定时间-电 流曲线时设定电压为 $0.05 \mathrm{~V}$. 在 $1.0 \mathrm{mmol} / \mathrm{L}$ $\mathrm{Fe}(\mathrm{CN})_{6}{ }^{3-/ 4-}+0.1 \mathrm{~mol} / \mathrm{L} \mathrm{KCl}$ 溶液中, 用 $\mathrm{CHI} 660 \mathrm{C}$ 电化 学分析仪记录各修饰电极的电化学交流阻抗(EIS) 曲 线, 应用电压 $5 \mathrm{mV}$, 外加电位为 $0.221 \mathrm{~V}$, 频率范围 为 $10^{-1} \sim 10^{4} \mathrm{~Hz}$.

\section{3 结果与讨论}

\section{1 双酶生物传感器的制备原理}

双酶生物传感器的制备过程如图 1 所示. GPTMS 的水解、缩合以及CS活性氨基与环氧基团的共价反 应已经通过固态核磁共振等证实 ${ }^{[10,11]}$. 与 CS 类似, 生 物酶HRP和GOD分子中具有活性氨基，与环氧基
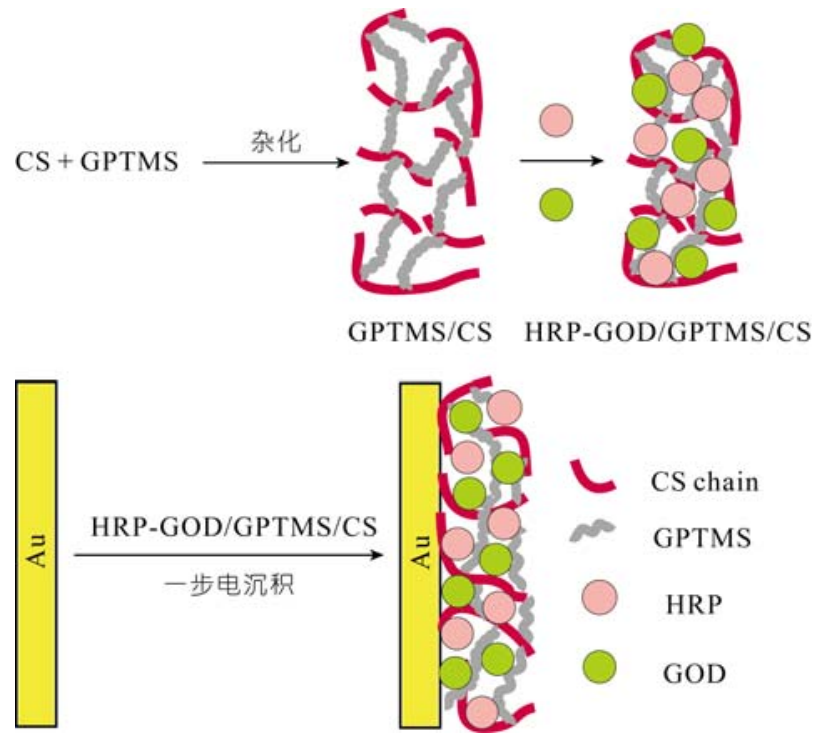

图 1 双酶生物传感器制备过程示意图 
团同样可发生共价反应. 在溶胶-凝胶过程中，一步 完成 CS、GPTMS 以及酶的共价交联与无机网络的引 入，从而得到交联型有机-无机杂化生物复合材料.

在 $\mathrm{Au}$ 电极表面原位电沉积可得到交联型有机-无 机生物杂化膜, 从而获得共固定 HRP和GOD的新型 双酶生物传感器. CS 电沉积法是利用CS酸溶碱不溶 的结构特点来实现的. 当以工作电极为阴极并施加 一定负电压时，溶液中 $\mathrm{H}^{+}$在阴极表面被还原成 $\mathrm{H}_{2} ，$ 以 致电极附近溶液 $\mathrm{pH}$ 值增加. $\mathrm{CS}$ 的 $\mathrm{p} K_{\mathrm{a}}$ 为 6.3 , 当 $\mathrm{pH}$ 大 于 6.3 时, CS 开始沉积在电极表面, 从而在电极表面

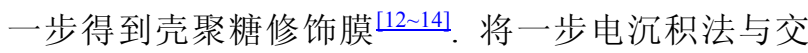
联型溶胶-凝胶反应相结合，在CS、GPTMS、HRP、 GOD混合溶液中进行电沉积，可在电极表面一步制 备 HRP-GOD/GPTMS/CS 生物杂化膜, 得到 HRP$\mathrm{GOD} / \mathrm{GPTMS} / \mathrm{CS} / \mathrm{Au}$ 修饰电极.

\section{2 双酶生物传感器的电化学表征}

GPTMS的用量是影响杂化膜稳定性的重要因素. 固定 $\mathrm{CS}(\mathrm{wt} \%)$ 的量为 $0.5 \%$, 考察不同 $\mathrm{GPTMS}(\mathrm{wt} \%)$ 用 量所得HRP-GOD/GPTMS/CS/Au对 $0.1 \mathrm{mmol} / \mathrm{L}$ 葡萄 糖 $\left(0.1 \mathrm{~mol} / \mathrm{L} \mathrm{PBS}+1.0 \mathrm{mmol} / \mathrm{L} \quad \mathrm{K}_{4}\left[\mathrm{Fe}(\mathrm{CN})_{6}\right]+0.1\right.$ $\mathrm{mol} / \mathrm{L} \mathrm{KCl}, \mathrm{pH} 4.0)$ 的长时间电化学响应，如图 2 所示. 当GPTMS用量小于 $1.0 \%$ 时，电极对葡萄糖的连续响 应很不稳定，电流响应随响应时间逐渐增大. 这可能 是由于GPTMS用量过低时, CS 杂化膜交联程度较低. 所以，酸性条件下电极表面未完全交联的 CS 杂化膜 逐渐溶解剥落, 使杂化膜的厚度减小, 电子传递电阻 $\left(R_{\mathrm{et}}\right)$ 值减小, 导致电流响应逐渐增大(曲线 1 3). 当 GPTMS 用量为 $1.5 \%$ 时 (曲线 4 ), 电极对葡萄糖的连续 响应十分稳定, 说明此时电极表面的 CS 杂化膜在酸 性条件下能够稳定存在. 这可能是由于 CS与 GPTMS 充分交联形成的有机一无机杂化膜，具有较强的耐 酸性，能够使电极对葡萄糖长时间连续测定而保持 电流稳定. 而当GPTMS用量进一步增加到 $2.0 \%$ 和 $2.5 \%$ 时，电流响应却随时间略有增大(曲线 5,6). 这 主要是由于GPTMS 用量较大时引入了大量功能性环 氧基团, 导致杂化膜具有较高的交联度. 尽管此时电 极表面的CS杂化膜在酸性条件下不会溶解剥落，但 高的交联度导致杂化膜致密性提高, 底物扩散减慢, 使得响应电流随时间略有增大. 因此，本文选择

\section{$1.5 \%$ 作为 GPTMS的使用量.}

电化学交流阻抗谱(EIS)是研究修饰电极表面特 征的有效工具. 图 3 是不同修饰电极在 $1.0 \mathrm{mmol} / \mathrm{L}$ $\left[\mathrm{Fe}(\mathrm{CN})_{6}\right]^{3-/ 4-}(1: 1)+0.1 \mathrm{~mol} / \mathrm{L} \mathrm{KCl}$ 溶液中测得的交 流阻抗谱图, 从图中可以看出, 不同修饰电极阻抗谱 图中电子传递阻抗 $\left(R_{\mathrm{et}}\right)$ 显著不同. 裸 $\mathrm{Au}$ 电极(曲线 1) 的 $R_{\mathrm{et}}$ 值为 $569 \Omega$, 而 $\mathrm{CS} / \mathrm{Au}$ (曲线 2)与 $\mathrm{GPTMS} / \mathrm{CS} / \mathrm{Au}$ (曲线 3 )的 $R_{\mathrm{et}}$ 值显著减小, 分别为 $85 \Omega$ 和 $167 \Omega$. 这

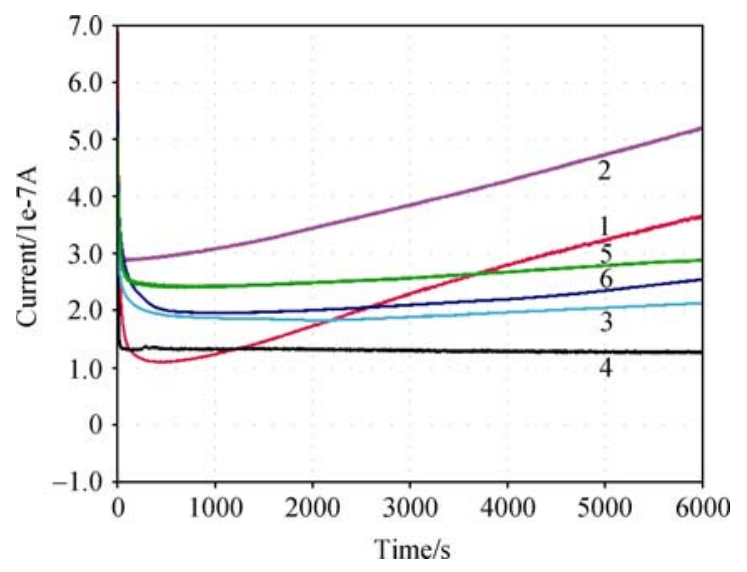

图 2 不同GPTMS(wt\%) 用量所得 HRP-GOD/GPTMS/ $\mathrm{CS} / \mathrm{Au}$ 修饰电极在 $1.0 \mathrm{mmol} / \mathrm{L} \mathrm{K}_{4}\left[\mathrm{Fe}(\mathrm{CN})_{6}\right]+0.1 \mathrm{~mol} / \mathrm{L}$ $\mathrm{KCl}+0.1 \mathrm{mmol} / \mathrm{L}$ 葡萄糖 $(0.1 \mathrm{~mol} / \mathrm{L} \mathrm{PBS}, \mathrm{pH} 4.0)$ 中的长时 间响应图

GPTMS 用量 $1 \sim 6$ 分别为 $0,0.5 \%, 1.0 \%, 1.5 \%, 2.0 \%, 2.5 \%$

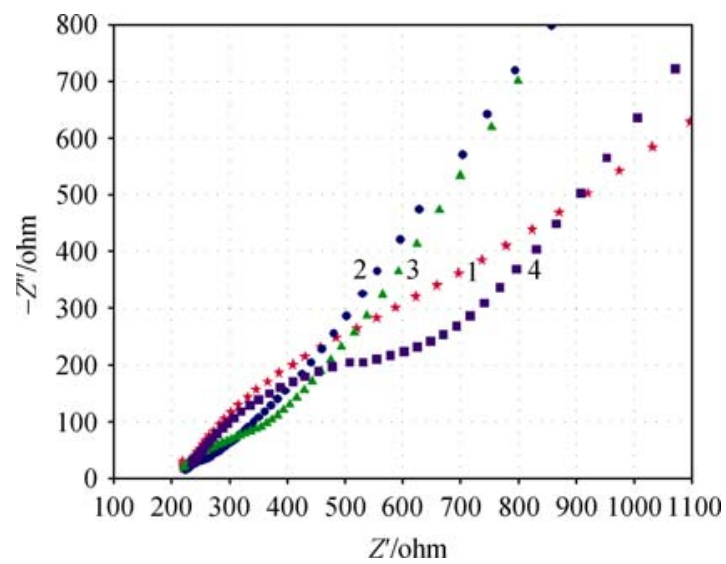

图 3 不同修饰电极在 $1.0 \mathrm{mmol} / \mathrm{L}\left[\mathrm{Fe}(\mathrm{CN})_{6}\right]^{3-/ 4-}(1: 1)+$ $0.1 \mathrm{~mol} / \mathrm{L} \mathrm{KCl}$ 溶液中的交流阻抗谱图

1 示裸 $\mathrm{Au}$ 电极; 2 示 $\mathrm{CS} / \mathrm{Au} ; 3$ 示 GPTMS/CS/Au; 4 示 HRP-GOD/ GPTMS/ CS/Au 
可能归因于电沉积杂化膜增大了电极的表面积而促 进了 $\mathrm{Fe}(\mathrm{CN})_{6}{ }^{3-/ 4-}$ 探针的电子传递. HRP-GOD/ $\mathrm{GPTMS} / \mathrm{CS} / \mathrm{Au}$ 电极(曲线 4 ) 的 $R_{\mathrm{et}}$ 值是为 $510 \Omega$, 高于 曲线 2 和 3 的 $R_{\mathrm{et}}$ 值, 表明杂化膜内负载了两种酶.

图 4 显示了裸 $\mathrm{Au}$ 电极 (曲线a)、HRP-GOD/ $\mathrm{GPTMS} / \mathrm{CS} / \mathrm{Au}$ (曲线b)在 $1.0 \mathrm{mmol} / \mathrm{L} \mathrm{K} \mathrm{K}_{4}\left[\mathrm{Fe}(\mathrm{CN})_{6}\right]+$ $0.1 \mathrm{~mol} / \mathrm{L} \mathrm{KCl}(0.1 \mathrm{~mol} / \mathrm{L} \mathrm{PBS}, 7.0)$ 中以及加入 0.1 $\mathrm{mmol} / \mathrm{L}$ 葡萄糖后修饰电极的响应(曲线c). 可以看出, 修饰电极的氧化还原峰电流高于裸 $\mathrm{Au}$ 电极, 而还原 峰与氧化峰电位基本不变. 对比加入葡萄糖前后修 饰电极的响应可以看出, 加入葡萄糖后还原峰电流 增大，氧化峰电流减小，这说明了电极表面的GOD对 葡萄糖产生催化反应, 其反应产物 $\mathrm{H}_{2} \mathrm{O}_{2}$ 又被HRP催 化, 整个反应机理如下:

$$
\begin{gathered}
\beta \text {-D-Glucose }+\mathrm{O}_{2} \stackrel{\text { GOD }}{\longrightarrow} \\
\text { D-Glucono-1,5-1 actone }+\mathrm{H}_{2} \mathrm{O}_{2} \\
\mathrm{H}_{2} \mathrm{O}_{2}+2 \mathrm{Fe}(\mathrm{CN})_{6}^{4-}+2 \mathrm{H}^{+} \stackrel{\text { HRP }}{\longrightarrow} \\
2 \mathrm{H}_{2} \mathrm{O}+2 \mathrm{Fe}(\mathrm{CN})_{6}^{3-}
\end{gathered}
$$

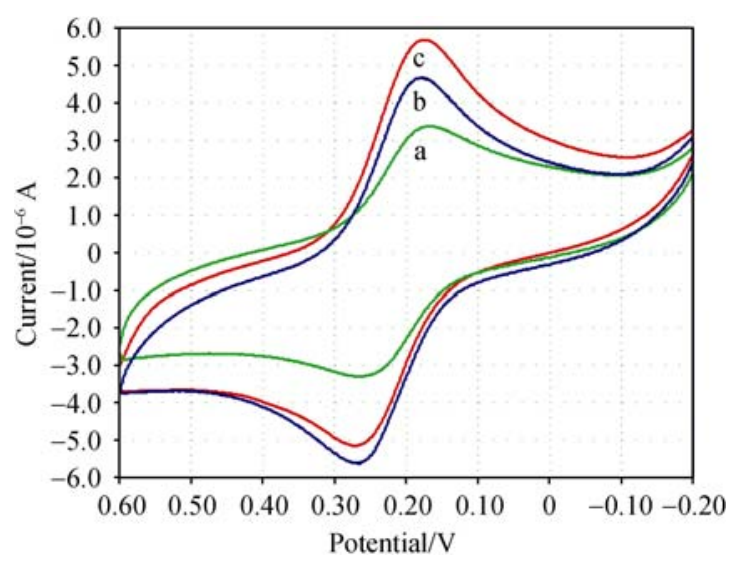

图 4 双酶生物传感器在 $1.0 \mathrm{mmol} / \mathrm{L} \mathrm{K} \mathrm{K}_{4}\left[\mathrm{Fe}(\mathrm{CN})_{6}\right]+0.1$

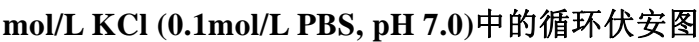
$\mathrm{a}$ 示裸 $\mathrm{Au}$ 电极; $\mathrm{b}$ 示 HRP-GOD/GPTMS/CS/Au, c 示 HRP-GOD/ $\mathrm{GPTMS} / \mathrm{CS} / \mathrm{Au}+0.1 \mathrm{mmol} / \mathrm{L}$ 葡萄糖

\section{3 双酶生物传感器在酸、碱溶液中的稳定性}

电极在不同酸、碱溶液中的稳定性十分重要. 分 别考察了 $\mathrm{pH} 4.0 、 7.0$ 和 10.0 的 $0.1 \mathrm{~mol} / \mathrm{L}$ PBS 溶液 中, 交联型 HRP-GOD/GPTMS/CS/Au 电极(曲线 a)与 未交联型 $\mathrm{HRP}-\mathrm{GOD} / \mathrm{CS} / \mathrm{Au}$ 电极 (曲线 b) 对 0.1 $\mathrm{mmol} / \mathrm{L}$ 葡萄糖连续响应的稳定性和耐用性，如图 5 所示. 显然, 在 $\mathrm{pH} 4.0$ 时, $\mathrm{HRP}-\mathrm{GOD} / \mathrm{CS} / \mathrm{Au}$ 电极电流 响应很不稳定．电流随时间变化显著增大，这说明电 极表面未杂化的壳聚糖膜不稳定, 发生溶解脱落. 然 而，尽管不同 $\mathrm{pH}$ 下酶活性差异会导致电流大小的差 别, 但交联型 $\mathrm{HRP}-\mathrm{GOD} / \mathrm{GPTMS} / \mathrm{CS} / \mathrm{Au}$ 电极在上述 三种 $\mathrm{pH}$ 条件下对葡萄糖的长时间(6000 s)连续电流 响应均保持稳定，说明 HRP-GOD/GPTMS/CS/Au 在 酸、碱溶液中均有良好的稳定性和耐用性.

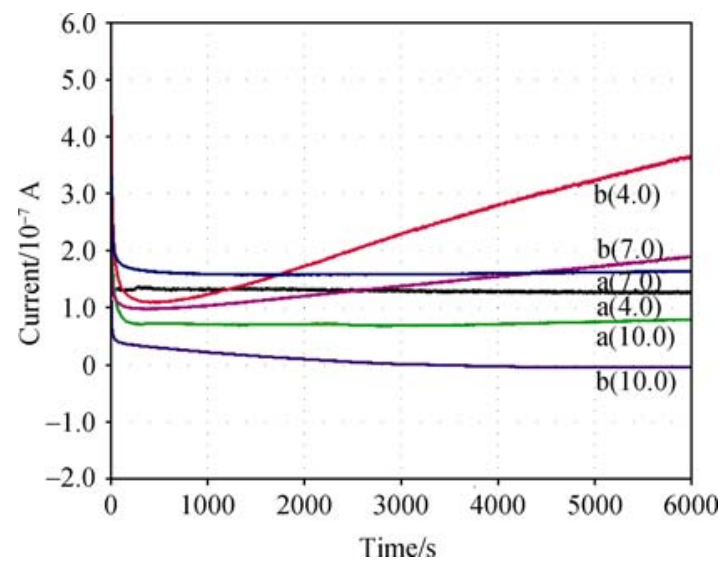

图 5 HRP-GOD/GPTMS/CS/Au (a) 和 HRP-GOD/CS/ $\mathrm{Au}(\mathrm{b})$ 电极在 $\mathrm{pH}$ 4.0, 7.0 和 $10.0 \mathrm{PBS}(0.1 \mathrm{~mol} / \mathrm{L}$, 含 1.0 $\left.\mathrm{mmol} / \mathrm{L} \mathrm{K} \mathrm{K}_{4}\left[\mathrm{Fe}(\mathrm{CN})_{6}\right]+0.1 \mathrm{~mol} / \mathrm{L} \mathrm{KCl}\right)$ 中对 $0.1 \mathrm{mmol} / \mathrm{L}$ 葡 萄糖的长时间响应图

\section{4 双酶生物传感器对葡萄糖响应的校准曲线}

图 6 是分别以HRP-GOD/GPTMS/CS/Au (1) 和 $\mathrm{HRP}-\mathrm{GOD} / \mathrm{CS} / \mathrm{Au}$ (2)为工作电极, 在摚动的 1.0 $\mathrm{mmol} / \mathrm{L} \mathrm{K}_{4}\left[\mathrm{Fe}(\mathrm{CN})_{6}\right]+0.1 \mathrm{~mol} / \mathrm{L} \mathrm{KCl}(0.1 \mathrm{~mol} / \mathrm{L} \mathrm{PBS}$, $\mathrm{pH}$ 7.0)中，连续加入不同浓度的葡萄糖所得到的电 流-时间曲线. HRP-GOD/GPTMS/CS/Au对葡萄糖的 电流响应很快, $7 \mathrm{~s}$ 内就能达到稳态电流的 $95 \%$. 与之 相比, HRP-GOD/CS/Au对葡萄糖的电流响应较慢. 内插图给出两种酶电极检测葡萄糖的标准曲线, HRP-GOD/GPTMS/CS/Au电极测定葡萄糖的线性范 围为 $1 \sim 351 \mu \mathrm{mol} / \mathrm{L}$, 检出限 $0.3 \mu \mathrm{mol} / \mathrm{L}$, 而未交联型 $\mathrm{HRP}-\mathrm{GOD} / \mathrm{CS} / \mathrm{Au}$ 测定葡萄糖的线性范围为 $1 \sim 41$ $\mu \mathrm{mol} / \mathrm{L}$. 与未交联型HRP-GOD/CS/Au相比，交联型 HRP-GOD/GPTMS/CS/Au电极具有更高的检测灵敏 度和更宽的线性范围。与文献报道的双酶传感器相 


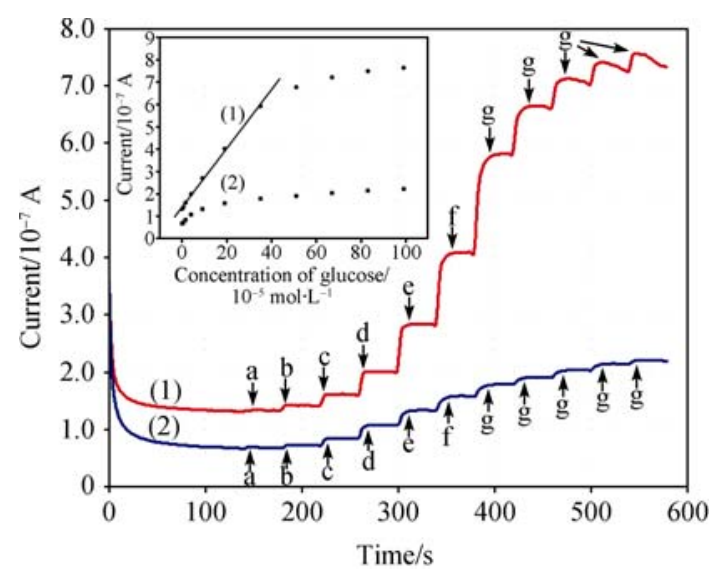

图 6 HRP-GOD/GPTMS/CS/Au (1) 和HRP-GOD/CS/Au (2) 在 $1.0 \mathrm{mmol} / \mathrm{L} \mathrm{K}{ }_{4}\left[\mathrm{Fe}(\mathrm{CN})_{6}\right]+0.1 \mathrm{~mol} / \mathrm{L} \mathrm{KCl}(0.1 \mathrm{~mol} / \mathrm{L}$ PBS, pH 7.0)中, 连续加入不同浓度葡萄糖的电流-时间 曲线

葡萄糖浓度 $\mathrm{a} \sim \mathrm{g}$ 分别为： $1,5,10,25,50,100,160 \mu \mathrm{mol} / \mathrm{L}$

比, HRP-GOD/GPTMS/CS/Au测定葡萄糖的检出限低 于 $\mathrm{HRP}-\mathrm{GOD} / \mathrm{Concanavalin} \mathrm{A} / \mathrm{CS} / \mathrm{Au}^{[15]} 、 \mathrm{GC} / \mathrm{CNT} /$ HRP-GOD/Nafion ${ }^{[16]}$ 和 $\mathrm{Au} / \mathrm{CNT} / \mathrm{HRP}-\mathrm{GOD} /$ polypy$\mathrm{rrole}^{[17]}$, 但高于 GC/CNT/HRP-GOD/thionine ${ }^{[18]}$.

\section{5 双酶生物传感器的重现性稳定性}

对 $100 \mu \mathrm{mol} / \mathrm{L}$ 葡萄糖进行 7 次平行测定的相对 标准偏差 $(\mathrm{RSD})$ 为 $2.7 \%$. 在同样的操作条件下, 考察 了不同电极的重现性，6根电极对 $100 \mu \mathrm{mol} / \mathrm{L}$ 葡萄糖 响应的RSD为 $3.1 \%$. 在 $4^{\circ} \mathrm{C}$ 下, 传感器保存六周后, 对 $100 \mu \mathrm{mol} / \mathrm{L}$ 葡萄糖的响应仍可达到原始响应的 $90 \%$, 说明传感器具备良好的稳定性. 这可能是由于 生物杂化膜具有良好的生物相容性导致 ${ }^{[9-11]}$.

\section{4 结论}

利用CS、HRP和GOD分子中- $\mathrm{NH}_{2}$ 与 GPTMS中环 氧基的共价偶联反应，经交联型溶胶-凝胶过程，在 $\mathrm{Au}$ 电极表面原位电沉积制备了交联型有机-无机生物 杂化膜, 一步得到共固定HRP和GOD的新型双酶生 物传感器. 证实了该交联型有机-无机生物杂化膜传 感器在不同酸、碱条件下都具有高的稳定性和耐用性, 克服了 $\mathrm{CS}$ 酸溶的不足，从而扩大了基于 $\mathrm{CS}$ 生物传感 器的使用范围。在葡萄糖的检测中, 交联型 HRP-GOD/GPTMS/CS/Au电极比无交联的HRP$\mathrm{GOD} / \mathrm{CS} / \mathrm{Au}$ 电极具有更高的灵敏度和更宽的线性 范围.

致谢本工作得到国家自然科学基金项目(批准号：20775039, 20775038)资助, 特此致谢.

\section{参考文献}

1 Coradin T, Allouche J, Boissiere M, Livage J. Sol-gel biopolymer/silica nanocomposites in biotechnology. Curr Nanosci, 2006, 2(3): $219-230$

2 Muzzarelli C, Muzzarelli R A A. Natural and artificial chitosan-inorganic composites. J Inorg Biochem, 2002, 92(2): 89—94[DOI]

3 Guibal E. Heterogeneous catalysis on chitosan-based materials: A review. Prog Polym Sci, 2005, 30(1): 71-109 [DOI]

4 Kaushik A, Khan R, Solanki P R, Pandey P, Alam J, Ahmad S, Malhotra B D. Iron oxide nanoparticles-chitosan composite based glucose biosensor. Biosens Bioelectron, 2008, 24 (4): 676-683 $\underline{\text { [DOI] }}$

5 Li F, Li X F, Zhang S S. One-pot preparation of silica-supported hybrid immobilized metal affinity adsorbent with macroporous surface based on surface imprinting coating technique combined with polysaccharide incorporated sol-gel process. J Chromatogr A, 2006, 1129 (2): $223-230 \underline{[\mathrm{DOI}]}$

6 Wang G H, Zhang L M. Using novel polysaccharide-silica hybrid material to construct an amperometric biosensor for hydrogen peroxide. J Phys Chem B, 2006, 110(49): 24864-24868[DOI]

7 Abdullah J, Ahmad M, Heng L Y, Karuppiah N, Sidek H. Stacked films immobilization of MBTH in nafion/sol-gel silicate and horseradish peroxidase in chitosan for the determination of phenolic compounds. Anal Bioanal Chem, 2006, 386(5): 1285-1292[DOI]

8 Tian X C, Li M J, Cai P X, Luo L J, Zou X Y. An amperometric cholesterol biosensor based on multiwalled carbon nanotubes and organically modified sol-gel/chitosan hybrid composite film. Anal Biochem, 2005, 337(1): 111-120[DOI]

9 Liu Y L, Su Y H, Lai J Y. In situ crosslinking of chitosan and formation of chitosan-silica hybrid membranes with using 
$\gamma$-glycidoxypropyltrimethoxysilane as a crosslinking agent. Polymer, 2004, 45(20): 6831—6837[DOI]

10 Liu Y L, Su Y H, Lee K R, Lai J Y. Crosslinked organic-inorganic hybrid chitosan membranes for pervaporation dehydration of isopropanol-water mixtures with a long-term stability. J Membr Sci, 2005, 251(1-2): 233-238[DOI]

11 Shirosaki Y, Tsuru K, Hayakawa S, Osaka A, Lopes M A, Santos J D, Fernandes M H. In vitro cytocompatibility of MG63 cells on chitosan-organosiloxane hybrid membranes. Biomaterials, 2005, 26(5): 485-493[DOI]

$12 \mathrm{Xu} \mathrm{J} \mathrm{J,} \mathrm{Luo} \mathrm{X} \mathrm{L,} \mathrm{Du} \mathrm{Y,} \mathrm{Chen} \mathrm{H} \mathrm{Y.} \mathrm{Application} \mathrm{of} \mathrm{MnO}_{2}$ nanoparticles as an eliminator of ascorbate interference to amperometric glucose biosensors. Electrochem Commun, 2004, 6(11): 1169-1173 [DOI]

13 Zhao G., Xu J J, Chen H Y. Fabrication, characterization of $\mathrm{Fe}_{3} \mathrm{O}_{4}$ multilayer film and its application in promoting direct electron transfer of hemoglobin. Electrochem Commun, 2006, 8(1): 148-154 [1DOI]

14 Xue M H, Xu Q, Zhou M, Zhu J J. In situ immobilization of glucose oxidase in chitosan-gold nanoparticle hybrid film on Prussian Blue modified electrode for high-sensitivity glucose detection. Electrochem Commun, 2006, 8(9): 1468 - 1474 [DOI]

15 Li F, Wang Z, Chen W, Zhang S S. A simple strategy for one-step construction of bienzyme biosensor by in-situ formation of biocomposite film through electrodeposition. Biosens Bioelectron, 2009, 24(10): 3030-3035[DOI]

16 Yao Y L, Shiu K K. A mediator-free bienzyme amperometric biosensor based on horseradish peroxidase and glucose oxidase immobilized on carbon nanotube modified electrode. Electroanalysis, 2008, 20(19): 2090-2095[DOI]

17 Zhu L, Yang R L, Zhai J L, Tian C Y. Bienzymatic glucose biosensor based on co-immobilization of peroxidase and glucose oxidase on a carbon nanotubes electrode. Biosens Bioelectron, 2007, 23(4): 528 - 535[DOI]

18 Jeykumari D R S, Narayanan S S. Fabrication of bienzyme nanobiocomposite electrode using functionalized carbon nanotubes for biosensing applications. Biosens Bioelectron, 2008, 23(11): 1686-1693 [DOD]

\title{
Construction of bienzyme biosensor based on combination of one-step electrodeposition and covalent-coupled sol-gel process
}

\author{
LI Feng ${ }^{*}$, WANG Zhen \& FENG Yan
}

Key Laboratory of Eco-Chemical Engineering, Ministry of Education, College of Chemistry and Molecular Engineering, Qingdao University of Science and Technology, Qingdao 266042, China

\begin{abstract}
Horseradish peroxidase (HRP) and glucose oxidase (GOD) bienzyme biosensor was constructed by in-situ formation of organic-inorganic biocomposite film based on one-step electrodeposition and covalent-coupled sol-gel process. The electrodeposition was performed in the solution containing functional inorganic precursor possessing epoxy group, $\gamma$-glycidoxypropyltrimethoxysiloxane (GPTMS), a biopolymer, chitosan (CS), and enzymes HRP and GOD. Covalent-coupled sol-gel process was formed by self-hydrolysis and self-condensation of GPTMS, and in-situ covalent cross-linking of CS, HRP, GOD through covalent reaction between amino groups and epoxy groups. The developed bienzyme biosensor presented high stability in acidic solution owing to the covalent-coupled organic-inorganic hybridization. Compared with non-hybrid HRP-GOD/CS/Au electrode, the bienzyme biosensor of HRP-GOD/GPTMS/CS/Au showed improved sensitivity and wider linear range for the determination of glucose. The linear response of the developed HRP-GOD/GPTMS/CS/Au biosensor for the determination of glucose ranged from $1 \mu \mathrm{mol} / \mathrm{L}$ to $351 \mu \mathrm{mol} / \mathrm{L}$ with a detection limit of $0.3 \mu \mathrm{mol} / \mathrm{L}$.
\end{abstract}

Keywords: chitosan, GPTMS, glucose, biosensor 